\title{
Organization And Methodology Laboratory Works On Biophysics For Dental Direction
}

\section{Nurmatova Feruza Bakhtiyarovna}

Tashkent Dental State Institute, Biophysics and information technology in medicine, Head of the chair, Tashkent, Uzbekistan. E-mail: feruzanurmatova_tdsi@mail.ru

\begin{abstract}
Quality medical education must be fundamental. An increase in the level of fundamentally of medical education should be associated with all-natural sciences, including the teaching of biophysics. The main goal of medical education is to improve the quality of training for the healthcare system based on high clinical, scientific, and ethical standards in medical education, the introduction, and the development of innovative educational technologies. At the same time, the basis of teaching should be the well-known didactic principles of pedagogy, and methods of teaching students the natural sciences - systematic, and consistent presentation of educational material, visualization, and accessibility, integration of sciences, and the spread of inter-subject relationships in the educational process at all its levels, requirements for a training experiment. The article shows the role of biophysics in a medical university, problems of teaching biophysics at the present stage, and some methods for solving them. Restore the formation of future dentists' skills to perform laboratory work of a professional nature, a technique is proposed for laboratory work.
\end{abstract}

Keywords:

biophysics, density, medical education, method, physical measurements, laboratory work, dentist, tooth.

Article Received: 18 October 2020, Revised: 3 November 2020, Accepted: 24 December 2020

\section{Introduction}

Biophysics is the science of the physical principles that underlie all the processes of life, including the dynamics, and kinetics of biological systems.

The subjects of biophysics are the physical principles that underlie all the processes of living systems. Biophysics is an interdisciplinary science somewhere between biology, and physics - as its name implies and, also, it is associated with other disciplines such as mathematics, physical chemistry, and biochemistry. Biophysics can be considered on an equal footing as part of physics. Especially today, when the boundaries between the classical disciplines are no longer established, it would be futile to try to balance these aspects with each other. Biophysics is one of the best examples of interdisciplinary science.

Medical, and biological physics helps to understand the meaning of physical phenomena occurring in the body, using physical laws in the diagnosis, and treatment of diseases. It defines the principles of the device, and operation of medical equipment teaches medical students to think logically, move from facts to concepts, build conclusions, enriching them with new modern knowledge.

Medical schools around the world are gradually moving from a traditional disciplinebased curriculum to an integrated one. Integrated training helps future doctors gather facts in a single chain to get a complete picture of the clinical situation and develop a holistic approach to treating a particular patient.

In the preparation of a dentist, the acquisition of basic theoretical knowledge in the basic fundamental disciplines taught in 1-2 courses of the dental institute is of great importance.

The training of a dentist in general practice, taking place at several specialized, and non-core departments of the dental institute, aims to instill in students the skills of continuous improvement, and effective application of knowledge for the analysis of clinical diseases, treatment methods, and predicting the effectiveness of their use. 
Our experience of working with students as dentists has revealed several points that complicate the implementation of this task, which is due to a very modest number of hours according to the curriculum; the unequal level of initial training in natural disciplines, and basic knowledge; the difference in personal characteristics, and target settings for training; a limited number of modern biophysics teaching aids for dentists.

Teaching the subject "Biophysics" provides classroom, laboratory, practical, and extracurricular methods.

Laboratory work and practical exercises are an important part of the theoretical, and vocational training of students. They are aimed at experimental confirmation of theoretical principles, and the formation of educational, and professional skills.

The main purpose of laboratory classes in physics is the acquisition by students of the necessary skills in conducting a physical experiment. At the same time, students should check the basic physical laws of phenomena, get acquainted with the measurement methods, and the rules for processing measurement results, and learn how to handle modern scientific equipment

Most measurements in medicine are measurements of physical or physicochemical quantities.

Measurements allow us to establish the laws of nature and are an element of knowledge of the world around us.

There are direct measurements, in which the result is obtained directly from measuring the quantity itself (for example, measuring the body temperature with a medical thermometer, measuring the length of an object with a ruler), and indirect, in which the desired value of the quantity is found from the known relationship between it, and the directly measured quantities (for example, the definition body weight when weighing, taking into account the buoyancy force, determining the viscosity of a liquid by the speed of falling of a ball in it). Technical means for making measurements (measuring instruments) can be of various types. The most famous measurement tool is a measuring device in which the measuring information is presented in a form that is accessible for direct observation by an observer (for example, the temperature is represented in a thermometer by the length of a column of mercury, current strength - by reading the ammeter arrow or a digital value). Measuring instruments also include a measure that is designed to reproduce a physical quantity of a given size (for example, a weight of a certain mass).

In practical classes in biophysics, all these types of measurements are actively used. For example,

1. Determination of the density of bodies of regular geometric shapes.

2. Determination of viscosity coefficient by the Stokes method.

3. Determination of surface tension coefficient by the drop separation method.

4. Determination of the wavelength of laser radiation using a diffraction grating, etc. [2].

In quantitative diagnostics - blood pressure, temporal dependence of biopotentials, the optical system of the eye, etc. In laboratory tests - blood viscosity, sugar concentration in urine, etc. During treatment, it is important to know the dose of ionizing radiation, current strength during galvanization, ultrasound intensity, etc.; the absence of any information of this kind can not only reduce the therapeutic effect but also cause harm. A quantitative assessment of the parameters of the environment surrounding a person (air humidity, temperature, atmospheric pressure) is a prerequisite for disease prevention, climate treatment.

All kinds of physical biomedical measurements can be classified either according to a functional attribute or by belonging to the corresponding section of physics. The physical classification is closer to the structure of this course, which is why it is given below. 
Mechanical measurements: anthropometric parameters of the body, movement, speed, and acceleration of body parts, blood, air, acoustic measurements, blood, and fluid pressure in the body, and air in the environment, vibration measurement, etc. Measurement devices: caliper, audiometer, tonometer, phonendoscope, psychrometer, ergometer, and others

Thermophysical measurements: temperature of organs, parts of the body, and the environment, calorimetric measurements of biological objects, food, etc. Instruments thermometer, calorimeter, etc.

Electrical, and magnetic measurements: biopotentials, induction of the magnetic field of the heart, measuring the impedance of biological objects, food, etc. Instruments: electrocardiograph, rheograph, etc.

Optical Measurements: colorimetric measurements, measurements of the optical characteristics of ophthalmic media for diagnostic purposes, spectral measurements for diagnostics, and forensic purposes, measurement of the characteristics of ultraviolet, infrared, and visible light for hygienic purposes, etc. Instruments: KFK-2 photo electro calorimeter, interferometers.

Atomic, and nuclear measurements: measurement of ionizing radiation, dosimetry, etc. Instruments: optical quantum generator, radiometers, dosimeters, radiometers.

In addition, you can specify physical, and chemical measurements: quantitative determination of the composition of the inhaled, and exhaled air, the gas composition of the blood, the $\mathrm{pH}$ of the blood, and other biological environments.

The functional principle of the classification of biomedical measurement methods can be shown by measuring the parameters of the cardiovascular system. Here there are mechanical (ballistic cardiograph, phonocardiography, blood pressure measurement), electrical, and magnetic (electrocardiography, magnetocardiography), optical measurements (oxyhemometry). Other physical methods are possible; for example, the method of nuclear magnetic resonance controls the speed of blood flow and more.

Laboratory research work is an exciting activity where students can not only test their knowledge but also gain practical experience.

The content of laboratory work is that the analysis verification of formulas, calculation directions, the establishment, and substation of laws, familiarization with the methods of experiments, the establishment of the properties of substances, their qualitative, and quantitative characteristics, observation, and development of phenomena, processes, etc. When choosing the content, and scope of laboratory work, it's necessary to proceed from: - the complexity of the tutorial material for assimilation, - intra-subject, and intra-subject communications, - what place a selected work occupies within the aggregate of laboratory work, and their significance for the formation of a holistic view of the content of the tutorial discipline. Varieties of organization of student add the laboratory, and practical classes: frontal, group, and individual. Within the frontal kind of the organization of classes, all students perform the identical work the identical time. Within the group type of organization of classes, the identical work is performed in groups of $2-5$ people. With a private sort of organization of classes, each student performs a personal task.

There are different tasks in the works. Each work has its own distinctive features, character, and structure. Illustrative - tasks are aimed at studying the external characteristics, the structure of the object in question. The work is performed in the form of diagrams, images, drawings, and diagrams. Research - students observe certain phenomena for a long time (weather changes, plant, and animal growth). Students record the results of the study in the form of graphs, numerical indicators, diagrams, and drawings. Summarizing - such work is carried out on the basis of the material passed to consolidate it. Problematic - during the course of work, the problem raised by the teacher is resolved. 
Practical - such work is carried out on the basis of theoretical knowledge for their application in practice.

Laboratory work biophysics for college students of medical institutes involves significant independent work both at the stage of preliminary preparation for work, and through the performance of labor, its design, and calculations.

For example, the work "Determining the ratio of a liquid employing a refract meter" can be set as follows. Within the first lesson, students learn the device and master the measurement method. The method is taken into account mastered if the ratio of the liquid is set with a slip of no more than $5 \%$ compared to the table value.

In the second lesson, you can study the dependence of the refractive index on the solution concentration in two ways:

1. Students are given solutions with a known concentration. Students measure refractive indices and plot $\mathrm{n}=\mathrm{f}$ (c). The refractive index of a solution of unknown concentration is measured, and the concentration is determined from the graph.

2. Each student receives a task to prepare a solution of a substance (for example, table salt) of a given concentration, and determine its refractive index. A table is crossed out on the board, and each student enters the results of their measurements into it. According to this table, students build a graph of the dependence $n=f(c)$. Inaccuracies in the work of individual students are clearly visible by the scatter of points on the graph. Some students may be instructed to take repeated measurements. The conclusion about the dependence of $\mathrm{n}$ on $\mathrm{s}$ is discussed. After that, each student receives a solution of the same substance of unknown concentration (each student has an individual task), measures the refractive index, and, according to the schedule, finds the concentration. Thus, the student gets acquainted with the refractometric method for determining the concentration of solutions.

Those students who quickly completed the task can familiarize themselves with the refractometric method for determining the percentage of protein in blood serum.

This method of work can be applied in other cases, when studying the surface properties of a liquid, studying the viscous properties of a liquid, etc.

All this increases the requirements for the level of physical, mathematical, and technical training of the future doctor, which is reflected in the current program on biophysics.

Let's give an example of laboratory work conducted with students' dental profiles.

Instruments, and accessories: technical scales, a set of weights, a wire for hanging a tooth, a bench for installing glass with liquid, a glass with distilled water, a measuring cylinder, adult teeth, a baby's baby tooth, and tooth teeth

\section{Theoretical part}

Density is a physical quantity determined for a homogeneous substance by its mass per unit volume (a value inverse to the specific volume of a substance). The density of an inhomogeneous substance is the ratio of mass, and volume when the latter is contracted to the point at which the density is measured. The ratio of the densities of two substances under certain standards of physical conditions is called relative density. For liquid, and solid substances it is measured at a temperature $t$, as a rule, in relation to the density of distilled water at $40 \mathrm{C}$, for gases in relation. The density of dry air or hydrogen under normal conditions $(\mathrm{T}=273.12, \mathrm{P}=1.01 *$ $105 \mathrm{~Pa})$. The density of substances usually decreases with increasing temperature (due to thermal expansion of bodies) and increases with increasing pressure. In the transition from one state of aggregation to another, the density is measured stepwise.

The mechanical strength and density of the tooth is ensured due to its optimal design, in which there is a high-strength thin surface layer enamel that protects bone tissue from the action of a "concentrated", point load, and enamel strength is provided at the ultra-, micro-, and macro levels. Bone tissue of a tooth - dentin has a denser structure than skeleton bones. Dentin, covered 
with a layer of enamel, through the dentin-enamel border perceives the external load on the tooth, forming a composite multilayer heterogeneous structure with enamel, which is a damper when the enamel is loaded.[1]

Teeth, along with the tongue, and the salivary glands, the ducts of which open into the oral cavity, serve as important organs for the mechanical processing, promotion, and digestion of food. Although in all vertebrates the teeth perform the same function of grinding food, their shape, and size are different and correspond to the kind of food consumed by these animals. In humans, the tongue, and teeth took on another function - the function of speech.

Despite the fact that externally the teeth can have a completely different look, their structure is basically the same. The part of the tooth protruding above the gum is called the crown, the part surrounded by the gum is the neck, and the part located to the bottom of the neck is the root. Each tooth consists of several layers: a hard outer layer or enamel, an inner layer (dentin), and a central cavity filled with pulp, in which there are blood vessels, and nerves. Enamel covers only the crown and the upper part of the neck of the tooth. A tooth is hardened in the jawbone by a substance called dental cement. Dentin in composition and hardness resembles bone, and consists of $72 \%$ of inorganic material, and $28 \%$ of organic matter. Enamel - the hardest substance in the body almost $97 \%$ consists of inorganic material.

Mammalian teeth are specialized for various functions. Eight front chisel teeth, called incisors, are used for biting. Four cone-shaped fangs, one in each front corner of the upper, and lower jaws, are used to tear food. Behind the fangs on each side of that, and the other jaw there are two pseudo-rooted teeth (premolar), and three molars (molars) with a flattened surface, adapted for crushing, and grinding food. Herbivores, for example horses, and cows, have wide, flat roots for grinding food, and developed incisors for nibbling grass. In humans, the teeth are relatively few specialized, since his ancestors were omnivores.
From the foregoing, it is clear that such parameters as hardness, density, strength are decisive for teeth. At the same time, due to the heterogeneity of the tooth structure, it is possible to speak about these parameters only in the average sense. Given the difference in the composition of food in different age periods, it seems interesting to compare the density of the tooth of an adult with a similar indicator of a child's milk tooth. From these positions, it is interesting to compare the tooth density of various representatives, for example, a person, and a horse.

\section{Research Method}

On everybody immersed in a liquid, a lifting force acts upward from the liquid side, and is applied to the center of gravity of the submerged body. The magnitude of this force is equal to the weight of the displaced fluid. In this formulation, although not in a very explicit form, gravity is assumed, since the existence of the buoyancy force is due to the difference in the statistical pressures in the liquid. An increase in the density of the liquid leads to an increase in the buoyancy force, and, consequently, to a decrease in the weight of the body immersed in the liquid. If the body is not completely immersed in the liquid, then moving the body deep into the liquid increases the buoyancy force. Methodhydrostatic weighing is as follows. The density of a solid is determined by double weighing - first in the air, then in a liquid whose density is known (distilled water). At the first weighing, body weight is determined, and the volume is determined by the difference in the results of both weighings. When measuring the density of a liquid, a body is weighed in it, the mass, and volume of which are known. Hydrostatic weighing, depending on the required density, is carried out on technical, analytical, or model scales. Since the strength of Archimedes is equal to the weight of the displaced fluid, knowing the density of the fluid at the test temperature (from the tables), we can determine its volume, which, in turn, is equal to the volume of a completely submerged body. Given the difference in the composition of food in different 
age periods, It seems interesting to compare the tooth density of an adult with a similar indicator of a child's milk tooth. From these positions, it is relevant to compare the tooth density of various representatives, for example, a person, and a horse.

\section{TASK №1}

\section{PERFORMANCE ORDER}

1. Weigh the wire for hanging the tooth.

2. Suspend the teeth being examined one by one using a wire to the balance beam, determine the mass, and subtract the mass of wire from it.

3. Place a bench with a glass of distilled water over the weighing pan, and lower the suspended tooth into it.

4. Determine the mass of weights balancing the body in water, and subtract the mass of the wire.

5. Calculate the density of the tooth under study by the formula:

$$
\rho 3=\rho \mathbf{B} \frac{m 1}{m 1-m 2}
$$

where $\mathrm{m} 1$ is the mass of the tooth in air, $\mathrm{m} 2$ is the mass of the tooth in water, $\rho v$ is the density of

water at a given temperature. The derivation of this formula is given below.

According to the law of Archimedes

$$
(\mathrm{m} 1-\mathrm{m} 2) \mathrm{g}=\rho \mathrm{BVg}(1)
$$

where $\mathrm{g}$ is the acceleration of gravity, $\mathrm{V}$ is the volume of the tooth is equal to: $\mathrm{V}=(2) \frac{\boldsymbol{m} \mathbf{1}}{\boldsymbol{\rho}_{3}}$

$$
\text { If we substitute (2) in (1) we have: }(\mathrm{m} 1-\mathrm{m} 2) \mathrm{g}=\rho \mathrm{B} g \frac{\boldsymbol{m} \mathbf{1}}{\boldsymbol{\rho}_{\mathbf{3}}}
$$

Record the data in a table.

Table1

\begin{tabular}{|c|c|c|c|}
\hline Teeth & $\begin{array}{c}\text { Mass in the air } \\
\mathrm{m} 1, \mathrm{~g}\end{array}$ & $\begin{array}{c}\text { Mass in water } \\
\mathrm{m} 2, \mathrm{~g}\end{array}$ & $\rho 3, \mathrm{~g} / \mathrm{cm} 3$ \\
\hline Horse & & & \\
\hline Person & & & \\
\hline Child & & & \\
\hline
\end{tabular}

\section{TASK №2}

\section{PERFORMANCE ORDER}

1. Weigh the teeth one by one and enter the $m$ (g) values in the table.

2. Pour distilled water into the graduated cylinder to the "10" mark.

3. Lower teeth alternately in the cylinder and each time determine the volume - V of water displaced by the tooth, for which subtract $10 \mathrm{ml}$ from the obtained values and enter the data in the table.

4. Calculate the density of the tooth under study by the formula $\rho_{3}=\frac{\boldsymbol{m}}{\boldsymbol{V}}$

5. Compare among themselves the values of the densities of the teeth of the horse, adult, and child

Table 2

\begin{tabular}{|c|c|c|c|}
\hline Teeth & $\begin{array}{c}\text { Tooth mass } \\
\mathrm{m}, \mathrm{g}\end{array}$ & $\begin{array}{c}\text { Tooth volume } \\
\mathrm{V}, \mathrm{cm} 3\end{array}$ & $\begin{array}{c}\text { Density } \\
\rho 3, \mathrm{~g} / \mathrm{cm} 3\end{array}$ \\
\hline Horse & & & \\
\hline Person & & & \\
\hline
\end{tabular}




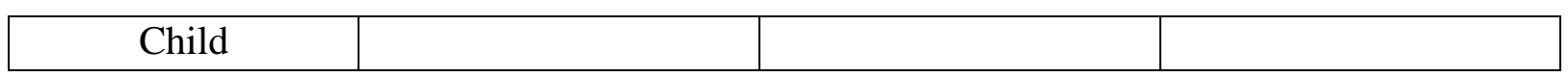

When finalizing the results, it should be noted that in these measurements, the errors are instrumented. The weighing error shall be considered equal to $0.005 \mathrm{~g}$; take the density of water with an accuracy of $0.1 \mathrm{~g} / \mathrm{cm} 3$. The error in determining the volume is $0.1 \mathrm{ml}$.

From the above, we found that such parameters as density are decisive for a number of representatives of the teeth. At the same time, due to the heterogeneity of the tooth structure, it is possible to speak about these parameters only in the average sense.

Based on our studies, it can be established that knowledge of the physical, and morphofunctional properties of hard tissues of teeth allows us to judge the ability of the dentofacial system to absorb loads, and not be destroyed. A qualitative assessment of the results of the study of tooth density showed their differences, which is due to different morphological, and functional properties, namely, the orientation of the protein-mineral skeleton of

\section{What is most interesting in biophysics lessons?}

\section{9 responses}

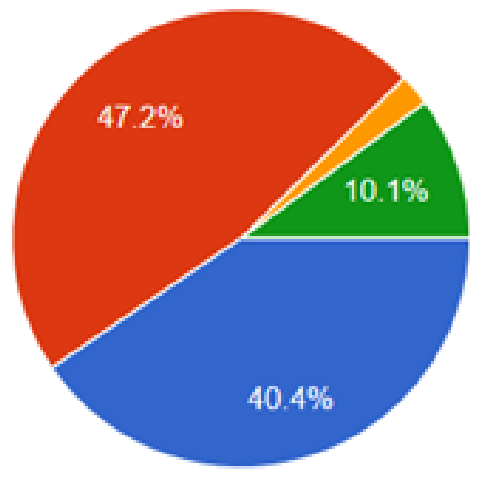

the tooth of various representatives in the longitudinal direction in accordance with the load necessary for chewing food.

The wear resistance of the working surface of hard tooth tissues, namely strength, and density, are closely related. The importance of the physical, and morphofunctional properties of hard tissues of the tooth allows a deeper understanding of the mechanisms of their work, as well as the conduct of new research in modern dentistry, which would significantly expand the indications for the use of adhesive systems of the restoration technique in dentistry.

After the end of the laboratory work, a survey was conducted among students. The essence of the questionnaire is to find out the students' opinions on the subject of biophysics. During the survey, 89 students participated. It turned out that $40.4 \%$ of the students like the teacher's explanation, $47.2 \%$ of the students like the lab work, and $10.1 \%$ of the students like to solve problems.
Listen to teacher's explanation

Perform laboratory work

Answer teacher's questions

To solve problems 


\section{The preferred form of work in the lesson for me is}

89 responses

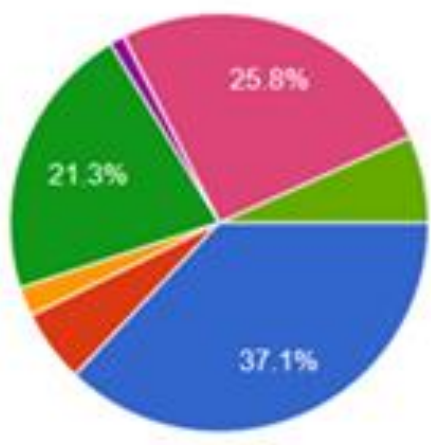

The survey shows that most students are interested in practical, and laboratory work, conversations on scientific topics, discussions, and explanations of teaching materials. Problemsolving makes it difficult for students.

\section{Conclusion}

Based on the analysis conducted by us in the laboratory, we can conclude:

- when performing such laboratory work, there is an opportunity for the synthesis of knowledge, the ability to transfer knowledge from one industry to another is being formed. This, in turn, stimulates the analytical activity of students, develops the need for a systematic approach to the object of cognition, and forms the ability to analyze, and compare complex processes, and phenomena of objective reality.

- integration of fundamental, and clinical subjects - a means of obtaining new ideas based on traditional subject knowledge. It is aimed at developing student erudition, at updating the existing narrow specialization in education;

- students will understand not only the presentation of the basic biophysical phenomena that underlie life, but also the well-known fundamental physical laws, and concepts. Under these conditions, it is possible to arouse interest in the subject and improve the assimilation of the material, along with the use of new pedagogical technologies, further bringing the questions outlined to the future specialty of students, profiling the subject, and shaping your biophysics.

\section{practical and research work}

independent study of material using a textbook, additional sources

Problem solving

teacher listening to explanation

others

listening to classmates

discussion, conversation on a scientific topic

a report to the group

\section{References}

[1] Bazarbayev M.I, Mullajonov I. And others Biophysics, Textbook 2018.

[2] Harten U. Physikfur Mediziner. - Springer, 2011.10.70-71s.

[3] P. Davidovits Physics in Biologt, and Medical. - 2013.- P 57-59.

[4] Roland Glaser Biophysics Springer Heidelberg Dordrecht London New York Library of Congress Control Number: 2012936486 \# Springer-Verlag Berlin Heidelberg 2012

[5] Biryukova A.N. (Kobzar, A.N.) Preparation for the solution of professional problems of medical students in physics studies taking into account interdisciplinary integration: diss. ... cand. ped Sciences: 13.00.02 [Text] / Biryukova Antonina Nikolaevna. - M., 2013.-- 277 p.

[6] Biryukova A.N. (Kobzar, A.N.) Physics at a medical university; under the editorship of S.I. Desnenko [Text] / A.N. Biryukova (A.N. Kobzar). - Chita: IITs ChGMA, 2012 .- 83 p.

[7] Nurmatova F.B., Kobzar A.N. Modern methods of physical properties of dental materials: Teaching aid - p.59

[8] The working curriculum on the subject "Biophysics". Field of education: 5510400 dentistry, 5511000 - pediatric dentistry. Tashkent: TGSI, 2019 .-- 23 p. 
[9] Rakhimova H.Zh., Nurmatova F.B. "Guideline for conducting practical classes in biophysics" 2018.

[10] Rakhimova Kh. Zh., Nurmatova F.B. The main physico-chemical properties of dental materials / Kh. Zh. Rakhimova, F.B. Nurmatova // Stomatologiya .- 2018. - No. 2.- p. 79

[11] Remizov, A.N. Medical, and biological physics: textbook 4th ed., Rev., and reslave. - M: GEOTAR-Media, 2018.

[12] T.A. Shirshova, T.A. PolyakovaLaboratory work as a means of motivating, and enhancing student learning. Omsk Scientific Herald № 4 (141) 2015 188-190c. 